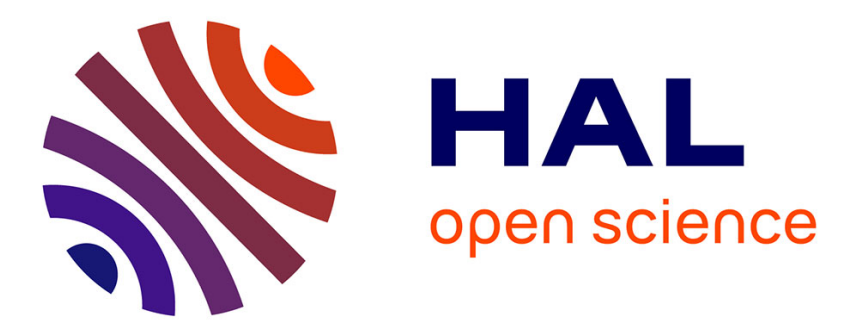

\title{
Angular studies of the magnetoresistance in the density wave state of the quasi two-dimensional purple bronze KMo6O17
}

\author{
H. Guyot, J. Dumas, M. Kartsovnik, J. Marcus, C. Schlenker, I. Sheikin, \\ David Vignolles
}

\section{To cite this version:}

H. Guyot, J. Dumas, M. Kartsovnik, J. Marcus, C. Schlenker, et al.. Angular studies of the magnetoresistance in the density wave state of the quasi two-dimensional purple bronze KMo6O17. The European Physical Journal B: Condensed Matter and Complex Systems, 2007, 58 (1), pp.25-30. 10.1140/epjb/e2007-00202-8 . hal-00202766

\section{HAL Id: hal-00202766 https://hal.science/hal-00202766}

Submitted on 8 Feb 2022

HAL is a multi-disciplinary open access archive for the deposit and dissemination of scientific research documents, whether they are published or not. The documents may come from teaching and research institutions in France or abroad, or from public or private research centers.
L'archive ouverte pluridisciplinaire HAL, est destinée au dépôt et à la diffusion de documents scientifiques de niveau recherche, publiés ou non, émanant des établissements d'enseignement et de recherche français ou étrangers, des laboratoires publics ou privés.

\section{(ㄷ)(1) $\$$}

Distributed under a Creative Commons Attribution - NonCommercial| 4.0 International 


\title{
Angular studies of the magnetoresistance in the density wave state of the quasi-two-dimensional purple bronze $\mathrm{KMo}_{6} \mathrm{O}_{17}$
}

\author{
H. Guyot ${ }^{1}$, J. Dumas ${ }^{1}$, M.V. Kartsovnik ${ }^{2}$, J. Marcus ${ }^{1}$, C. Schlenker ${ }^{1,5, a}$, I. Sheikin ${ }^{3}$, and D. Vignolles ${ }^{4}$ \\ 1 Institut Néel, CNRS/UJF- BP 166, 38042 Grenoble Cedex 9, France \\ 2 Walther-Meissner-Institut, Bayerische Akademie der Wissenschaften, Walther-Meissner-Strasse 8, 85748 Garching, Germany \\ 3 Grenoble High Magnetic Field Laboratory, GHMFL, BP 166, 38042 Grenoble Cedex 9, France \\ 4 Laboratoire National des Champs Magnétiques Pulsés, LNCMP 143 Avenue de Rangueil, 31077 Toulouse, France \\ ${ }^{5}$ Institut National Polytechnique de Grenoble, ENSPG, BP257, 38016 Grenoble Cedex 1, France
}

\begin{abstract}
The purple molybdenum bronze $\mathrm{KMo}_{6} \mathrm{O}_{17}$ is a quasi-two-dimensional compound which shows a Peierls transition towards a commensurate metallic charge density wave (CDW) state. High magnetic field measurements have revealed several transitions at low temperature and have provided an unusual phase diagram "temperature-magnetic field". Angular studies of the interlayer magnetoresistance are now reported. The results suggest that the orbital coupling of the magnetic field to the CDW is the most likely mechanism for the field induced transitions. The angular dependence of the magnetoresistance is discussed on the basis of a warped quasi-cylindrical Fermi surface and provides information on the geometry of the Fermi surface in the low temperature density wave state.
\end{abstract}

\section{Introduction}

The so-called purple bronze $\mathrm{KMo}_{6} \mathrm{O}_{17}$ belongs to the class of the low dimensional conducting transition metal oxide bronzes which show anisotropic Fermi surfaces and therefore CDW instabilities [1-3]. Among these materials, $\mathrm{KMo}_{6} \mathrm{O}_{17}$ is a quasi two-dimensional (2D) metal due to a trigonal layered structure including $\mathrm{MoO}_{6}$ octahedra layers separated by $\mathrm{K}^{+}$ions and $\mathrm{MoO}_{4}$ tetrahedra $[4,5]$. The conduction electrons are confined in the infinite layers perpendicular to the $c$-axis, leading to quasi-2D electronic properties. Electrical resistivity and magnetic susceptibility studies, followed by crystallographic investigations, have established that a Peierls instability occurs at $T_{P}=110 \mathrm{~K}$ towards a metallic commensurate charge density wave state. This is due to openings of partial gaps on the quasi-cylindrical Fermi surface. Band structure calculations with a $2 \mathrm{D}$ tight binding method lead to three partially filled bands crossing the Fermi level [6]. They correspond to three quasi-1D Fermi surface sheets related to zig-zag $\mathrm{MoO}_{6}$ octahedra chains along the $a, b$ and $(a+b)$ directions. This is the so-called hidden nesting property. These band calculations predict nesting wave vectors $(a * / 2,0,0)$ and equivalent, which have been observed by electron diffraction and X-ray diffuse scattering $[7]$.

\footnotetext{
a e-mail: claire.schlenker@grenoble.cnrs.fr
}

Band structure studies have been performed by angleresolved photoemission spectroscopy (ARPES) at room temperature. Fermi surface (FS) imaging performed with the same technique has corroborated the hidden nesting model $[8,9]$. Further experiments have explored the temperature dependence of the band structure with the opening of the Peierls gap [10]. All explored physical properties correspond to a CDW gap opening at $110 \mathrm{~K}$ with a doubling of the unit cell in the $(a, b)$ plane. STM studies establish that the CDW modulation takes place simultaneously along the three equivalent directions $a, b$ and $-(a+b)$; this corresponds to a triple- $q(2 a \times 2 b)$ hexagonal superstructure, since no domain structure with modulation along one of these three directions is observed by STM. This also corroborates that the trigonal symmetry is kept in the CDW phase [11].

However the nature of the low temperature state $(T<$ $30 \mathrm{~K}$ ) is not clear. The magnetic susceptibility shows an anomaly at approximately $25 \mathrm{~K}$ and an anisotropy suggesting an antiferromagnetic-type behaviour [4]. The curves of the magnetization vs. magnetic field show at $4.2 \mathrm{~K}$ a bending in the range of $2.5 \mathrm{~T}$ also consistent with such a behaviour [12]. One cannot exclude, therefore, the existence of a SDW or of a mixed SDW/CDW ground state.

In this density wave (DW) state, the in-plane magnetoresistance $\Delta \rho / \rho_{0}$ measured with the magnetic field perpendicular to the layers has been found positive and 


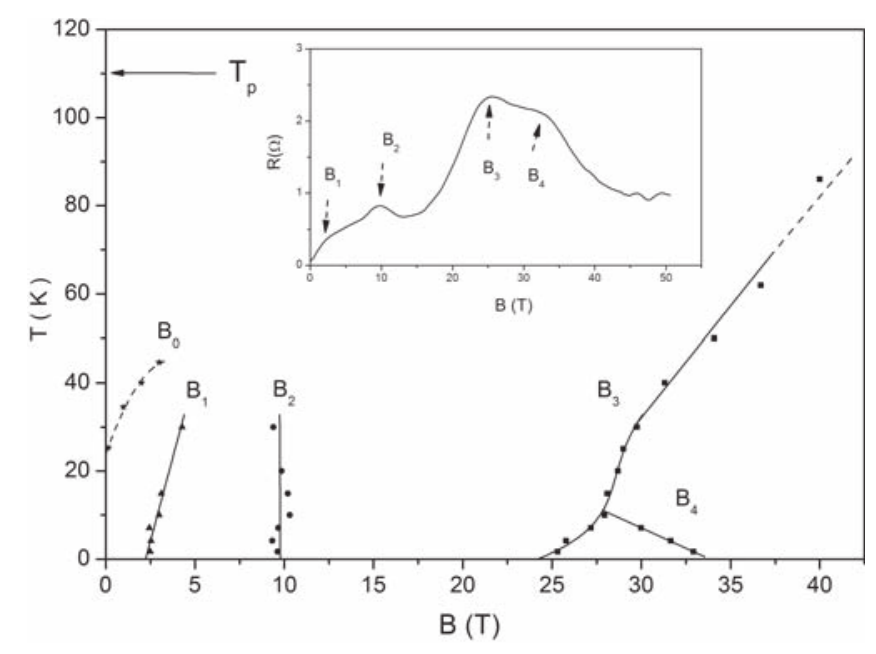

Fig. 1. Phase diagram showing the field positions $B_{i}$ of the anomalies and transitions for several temperatures (Ref. [17]). The Peierls temperature $T_{p}$ is also indicated. The line $B_{0}$ of a possible density wave transition is also shown (Ref. [13]). The insert shows the resistance vs. field at $T=1.3 \mathrm{~K}$ (Ref. [17]).

very large. This has been attributed to the coexistence of hole and electron pockets with high mobilities on the Fermi surface of the DW state. Detailed studies of the temperature dependence of this magnetoresistance supported a low temperature low field $(B, T)$ phase diagram showing a phase transition at $T \sim 25 \mathrm{~K}$ in zero field [13]. This has been corroborated by more recent magnetoresistance measurements performed on the three compounds $\mathrm{KMo}_{6} \mathrm{O}_{17}, \mathrm{TlMo}_{6} \mathrm{O}_{17}$ and $\mathrm{NaMo}_{6} \mathrm{O}_{17}$ [14]. Tunnelling studies presently on progress could confirm this model [15].

High magnetic field studies performed at low temperatures $(T=1.6 \mathrm{~K})$ with pulsed fields perpendicular to the layers, showed on the interlayer magnetoresistance curves $\Delta \rho / \rho_{0}$ vs. field several anomalies, followed by an anomalous decrease of the magnetoresistance above $30 \mathrm{~T}$ (see insert of Fig. 1). A change of slope is found in the vicinity of $B_{1}=2 \mathrm{~T}$, a maximum at $B_{2}=9.4 \mathrm{~T}$ and two other maxima at $B_{3}=24.5 \mathrm{~T}$ and $B_{4}=31 \mathrm{~T}$ [16]. A phase diagram $(T, B)$, including a first order transition in the range of $30 \mathrm{~T}$, has been proposed on the basis of these data [17] (Fig. 1). This phase diagram includes two lines at low fields, weakly dependent on temperature. The two first order transitions found at $1.6 \mathrm{~K}$ at high fields merge into a single one above $10 \mathrm{~K}$. The Peierls transition at $T_{p}=110 \mathrm{~K}$, is also shown on this diagram. $T_{p}$ is found field independent up to $30 \mathrm{~T}$ within the experimental accuracy $( \pm 2 \mathrm{~K})$. The line $B_{o}$ corresponds to results obtained previously showing another anomaly of the resistivity vs. temperature in low fields (line $T_{A}$ in Ref. [13]).

Further studies down to $150 \mathrm{mK}$, in a field up to $15 \mathrm{~T}$, have established that this phase diagram is valid down to very low temperature [33]. They also corroborate that the anomalies found on the curves $\rho(B)$ are not due to quantum oscillations.

A strong magnetic field is expected to influence the CDW state via the orbital and the Pauli paramagnetic mechanisms $[18,19]$. Field induced transitions associated with these mechanisms indeed have been observed on the organic conductors $\alpha$-(BEDT-TTF $)_{2} \mathrm{MHg}(\mathrm{SCN})_{4} \quad(\mathrm{M}=$ $\mathrm{K}, \mathrm{Rb}, \mathrm{Tl}$, or $\mathrm{NH}_{4}$ ) [20-23]. In particular, the so-called kink transition in those compounds associated with the Pauli effect $[21,22]$ strongly resembles the $B_{3}-B_{4}$ anomaly in $\mathrm{KMo}_{6} \mathrm{O}_{17}$. Thus, the present Mo purple bronze is, to the best of our knowledge, the first quasi-2D inorganic material showing in the CDW state the same type of high-field properties as the organic salts.

To gain further insight into the origin of the field induced transitions in this compound we have performed detailed studies of its magnetoresistance as a function of field orientation in high magnetic field at low temperature. Additionally, these experiments were expected to provide valuable information about FS parameters in this quasi2D material [24,25].

\section{Experiment}

Single crystals used in these studies have been grown by electrolytic reduction of a $\mathrm{K}_{2} \mathrm{CO}_{3}-\mathrm{MoO}_{3}$ melt. They have the shape of small platelets, typically $3 \times 1 \times 0.2 \mathrm{~mm}^{3}$, with the plane perpendicular to the $c$-axis [4].

Interlayer magnetoresistance measurements have been performed at Grenoble High Magnetic Field Laboratory (GHMFL) in dc fields up to $28 \mathrm{~T}$. The magnetoresistance has been measured at a temperature of $1.8 \mathrm{~K}$, using a standard four wire method and ac low frequency current perpendicular to the 2D layers. In order to study the angular dependence of the magnetoresistance, the sample was mounted on a two-axis rotator in a $\mathrm{He}$ cryostat. It was possible to change continuously in-situ the polar angle $\theta$ between the magnetic field and the normal to the plane of the layers (trigonal $c$-axis of the crystal) and to change discretely the azimuthal angle $\phi$ between the projection of the field on the plane and the crystallographic $a$-axis. The initial orientation of the sample was set to an accuracy of about $\pm 2^{\circ}$ under a microscope; during the measurements the angles $\theta$ and $\phi$ were varied with a resolution better than $0.1^{\circ}$ between $-120^{\circ}$ and $+120^{\circ}$ and between $0^{\circ}$ and $180^{\circ}$ respectively.

Figure $2 \mathrm{a}$ shows the field dependence of the magnetoresistance at $T=1.8 \mathrm{~K}$ in the field range $0-28 \mathrm{~T}$ for different angle $\theta$ between $0^{\circ}$ and $90^{\circ}$ for $\phi=0^{\circ}$. Three anomalies are clearly seen at $B_{1}=2.3 \mathrm{~T}, B_{2}=9.1 \mathrm{~T}$ and $B_{3}=24.4 \mathrm{~T}$ for $\theta=0^{\circ}$, as previously reported [17]. When $\theta$ is increased, these anomalies are displaced towards higher fields and their amplitude decreases. For $\theta=75^{\circ}$, they have nearly vanished (Fig. 2a). Figure $2 \mathrm{~b}$ shows the same data, plotted as a function of $B \cos \theta$. For low values of $\theta$, roughly smaller than $55^{\circ}$, the curves nearly coincide with each other, showing that the magnetoresistance depends mainly on the component of the field perpendicular to the layers. One should note that for $\theta$ larger than $55^{\circ}$, the field dependence changes. For $\theta=90^{\circ}$ the magnetoresistance is approximately proportional to $B$ and shows no anomaly. For $\theta<55^{\circ}$ the positions of the anomalies $B_{i}$ also scale to the perpendicular 


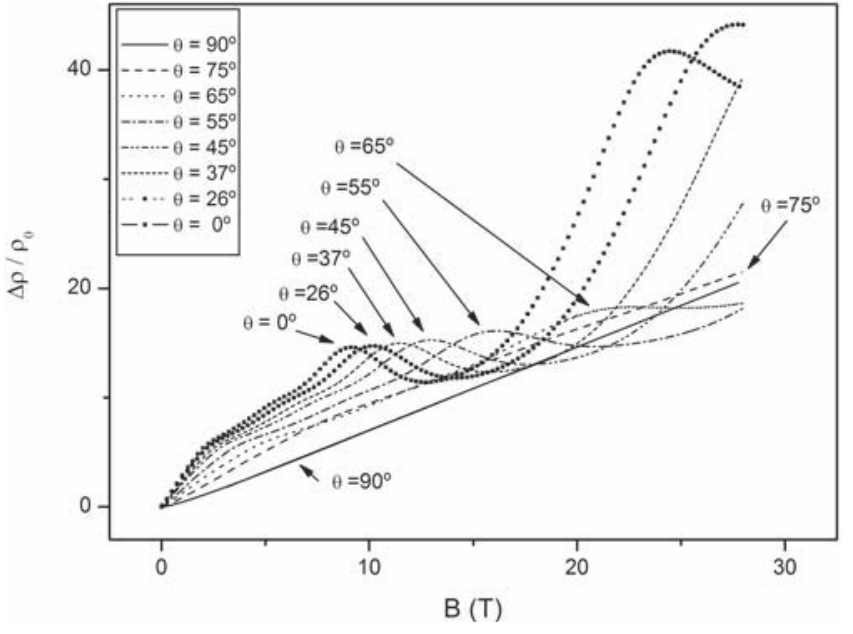

(a)

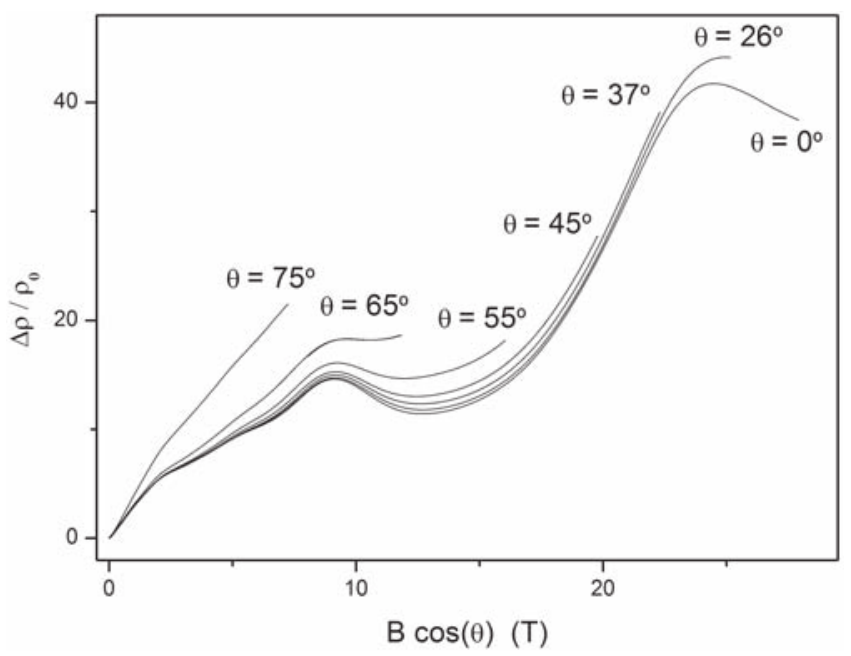

(b)

Fig. 2. (a) Magnetoresistance vs. dc magnetic field for different values of the angle $\theta$ between the field and the normal to the layers. $T=18$ K. (b) Same as in (a) plotted as a function of the field component normal to the layers.

field component as seen from Figure 2b. This behaviour is fully consistent with the results obtained in pulsed magnetic fields [26], as shown in Figure 3, since the angular dependence of the normalized magnetic fields $B_{i}$ follows a $1 / \cos \theta$ law.

The resistance measured vs. $\theta$ in dc fields at $T=1.8 \mathrm{~K}$ is shown in Figure $4 \mathrm{a}$ for different values of $B$ between 8 and $26 \mathrm{~T}$. The azimuthal angle was $\phi=0^{\circ}$. One notes a change of behaviour around $\theta=0^{\circ}$ when $B$ is increased, from a maximum to a local minimum and finally two maxima at higher field. For large values of $\theta$, close to $90^{\circ}$, complicated structures with several peaks appear. Figure $4 \mathrm{~b}$ shows the same data plotted vs. $B \cos \theta$. The superposition of the curves at large $B \cos \theta$ indicates that the $\theta$ dependence around $\theta=0^{\circ}$ is related to the $\cos \theta$ law as shown in Figures $2 \mathrm{~b}$ and 3 . For $B \cos \theta<18 \mathrm{~T}$, the curves progressively separate and several structures appear. A new behaviour appears for $\theta$ close to $90^{\circ}$. This is reflected

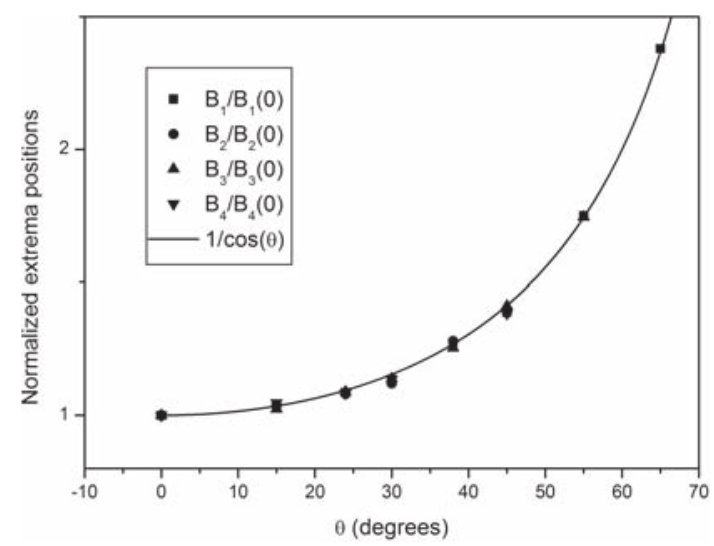

Fig. 3. Normalized magnetic field positions of the anomalies $B_{1} B_{2}, B_{3}, B_{4}$ vs. the polar angle $\theta$. Solid line is a fit according to a $1 / \cos \theta$ law. $T=2.2 \mathrm{~K}$ (Ref. [26]).

in the smooth linear dependence of the resistance vs. $B$ for $\theta=90^{\circ}$ (see Fig. 2a). Such a dependence can be ascribed to the normal magnetoresistive effect in a quasi-2D metal $[27,28]$. The splitting of the curves into two for $\theta$ close to $90^{\circ}$, corresponds to the fact that $R(B, \theta)$ differs from $R(B,-\theta)$. Whether this is due to a slight misorientation of the sample or to a basic mechanism is not clear at this point.

Figure 5a shows the curves $R(\theta)$ obtained for different values of the azimuthal angle $\phi$ at $B=19$ T. Figure 5b shows an enlargement of the features found around $\theta=90^{\circ}$. Two behaviours are observed, depending on the value of $\theta$. For $-68^{\circ}<\theta<75^{\circ}$, the resistance does not depend on $\phi$, whereas it does for $75^{\circ}<\theta<112^{\circ}$. In particular, the value of the resistance obtained in the vicinity of $\theta=90^{\circ}$, when $B$ is nearly parallel to the layer plane, depends strongly on $\phi$ : this is shown in Figure 6a on a polar diagram. This exhibits a sixfold symmetry consistent with the crystal symmetry.

\section{Discussion}

We will first analyze the phase diagram before discussing the angular dependence of magnetoresistance. As mentioned before, it is likely that the line $B_{0}$ corresponds to a transition from the commensurate CDW to another density wave state. This density wave state should include a spin component, as indicated by magnetic susceptibility data [4]. Tunnelling studies [15] as well as high resolution specific heat measurements [29], presently on progress, could corroborate this picture.

The anomalies corresponding to lines $B_{1}, B_{2}$ and $B_{3}$ are not periodic in the inverse field scale and, therefore, cannot be ascribed to the Shubnikov-de Haas effect. We rather propose that the anomaly found at $B_{1}$, which is a change of slope on the curve of the magnetoresistance vs. field, is due to a spin rotation as indicated by the curves of the magnetization vs. field [12].

Figures 2-4 show that positions of all these anomalies depend only on the $B$ component perpendicular to the 


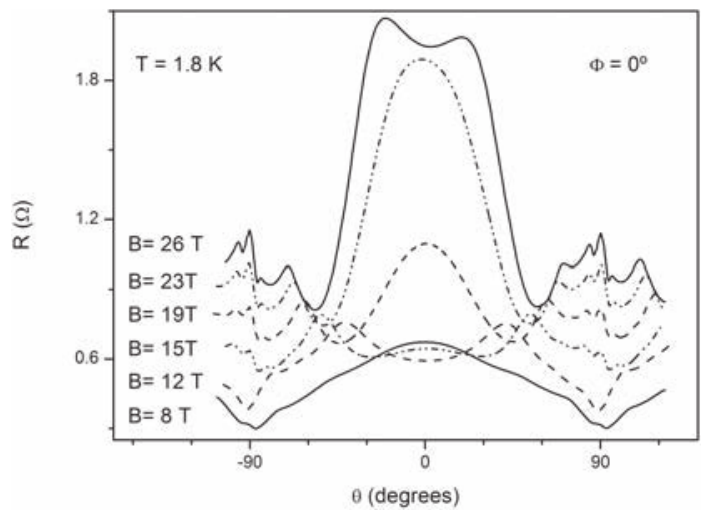

(a)

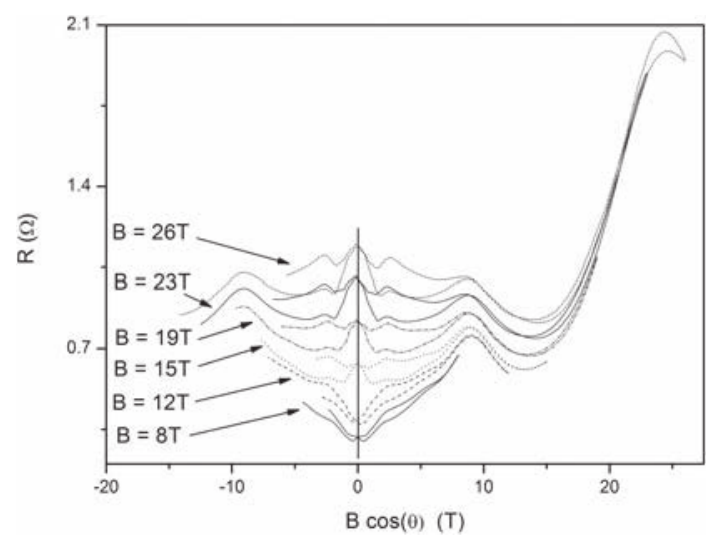

(b)

Fig. 4. Resistance vs. $\theta$ between $-120^{\circ}$ and $+120^{\circ}$ for different values of $B$. Azimuthal angle $\phi=0^{\circ} . T=1.8 \mathrm{~K}$. (b) Same data plotted as a function of $B \cos \theta$.

layers. This result indicates that the effect of the magnetic field is mainly due to orbital effects and that the Pauli spin contribution is not important.

In this context, the lines $B_{2}$ and $B_{3}$ may correspond to field induced transitions as studied previously in the spin density wave (TMTSF) $)_{2} \mathrm{X}$ organic salts $[30,31]$. These transitions are attributed to quantum nesting conditions on the Fermi surface: they correspond to an adaptation of the nesting vector on the quantized Fermi surface, such that a carrier pocket will contain an integer number of Landau levels. A similar cascade of field-induced transitions is predicted by theory also for CDW systems [19] and, indeed, has been reported for $\alpha$-(BEDT$\mathrm{TTF})_{2} \mathrm{KHg}(\mathrm{SCN})_{4}$ [23]. One can therefore expect the anomalies in the present compound to be related to the same phenomenon.

Angular studies of the magnetoresistance have been performed at low temperature $(T=1.8 \mathrm{~K})$ in the low temperature density wave state. The curves of $\Delta \rho / \rho_{o}$ vs. $B$ for different polar angles $\theta$ (Figs. 2a and $2 \mathrm{~b}$ ) establish that for $\theta$ smaller than $\cong 55^{\circ}$, the properties are due to the anomalies found at $B_{1}, B_{2}$ and $B_{3}$ for $\theta=0$, since the curves plotted vs. $B \cos \theta$ are approximately superimposed. This shows that only the component of the field perpendicular to the layers is active in this range of $\theta$. New phenomena

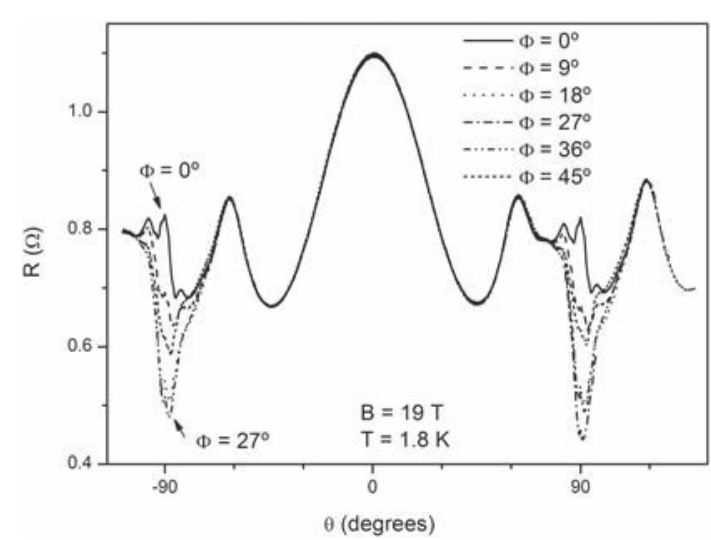

(a)

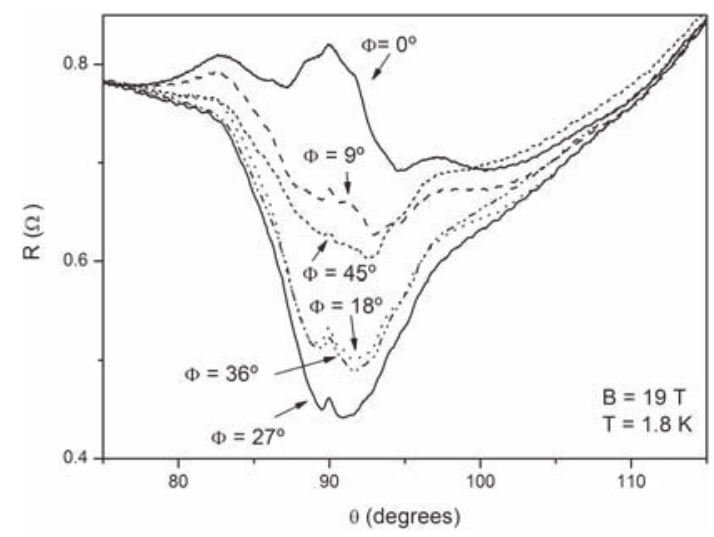

(b)

Fig. 5. Resistance vs. $\theta$ between $-120^{\circ}$ and +120 for different values of $\phi . B=19 \mathrm{~T}, T=1.8 \mathrm{~K}$. (b) Same as (a) showing the curves around $\theta=90^{\circ}$.

occur only for larger values of $\theta$. Analysis of the curves of the resistivity vs. $\theta$ for different values of $B$ leads to similar conclusions. Figure $4 \mathrm{~b}$ shows the data of Figure $4 \mathrm{a}$ plotted as $R$ vs. $B \cos \theta$. It appears that for values of $B \cos \theta$ larger than $\cong 8 \mathrm{~T}$ the curves are very similar. In this region, variations between the curves as well as the asymmetry of the maxima found at small angles for $B=26 \mathrm{~T}$ might be due to a small misorientation of the crystal. This shows again that for small values of $\theta$ (large values of $B \cos \theta$ ), only the component of $B$ perpendicular to the layers is active. Such a behaviour is in a striking contrast to what is usually observed in layered metals. Extensive studies of magnetotransport phenomena in organic conductors have shown that the interlayer magnetoresistance of a quasi2D metal essentially depends on both field components, perpendicular and parallel to conducting layers [24,25]. In particular, depending on the FS topology, various kinds of angular magnetoresistance oscillations (AMRO) emerge, whose positions are periodic in the scale of $\tan \theta$ and do not depend on the field strength. By contrast, the lowtemperature magnetoresistance of the present compound is insensitive to the inplane field component at $|\theta| \leqslant 55^{\circ}$ and all the features in this angular range are simply scaled to the anomalies observed in the perpendicular field. As $\theta$ approaches $90^{\circ}$, the magnetoresistance turns to be more 


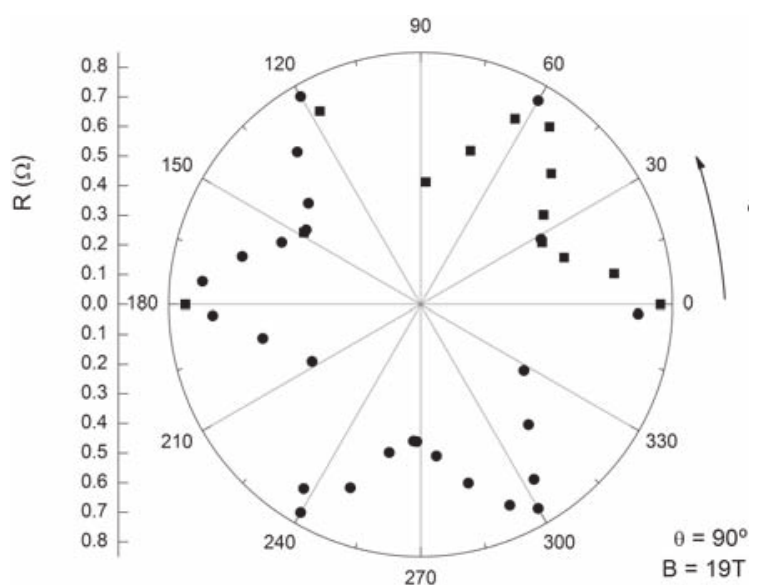

(a)

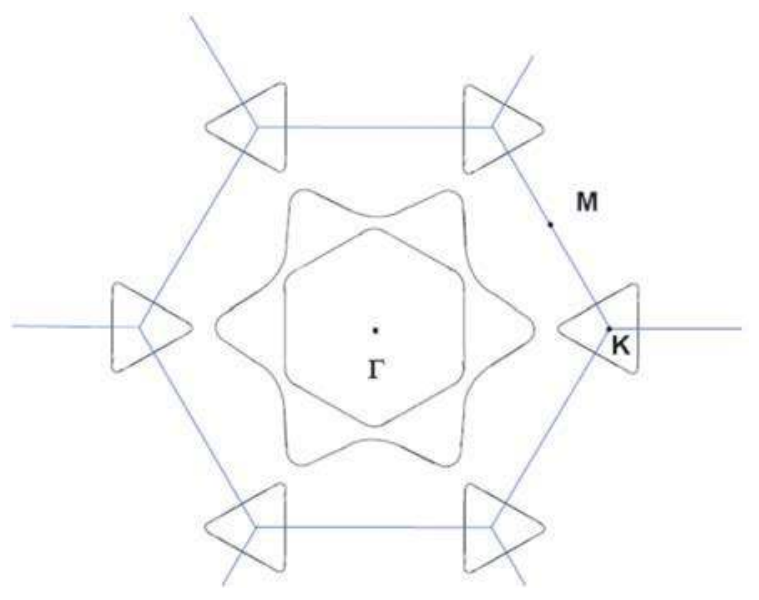

(b)

Fig. 6. Resistance at $\theta=90^{\circ}$ is a function of $\phi$, (square dots) on a polar diagram. The original measured data are represented by square dots; the circles have been obtained from the original data by threefold rotations. $B=19 \mathrm{~T}, T=1.8 \mathrm{~K}$. (b) Calculated Fermi surface of $\mathrm{KMo}_{6} \mathrm{O}_{17}$ in the normal high temperature state (solid line) (from Ref. [6]).

conventional, being now sensitive to both the strength and orientation of the inplane field component, see Figures $4 \mathrm{~b}$ and 5. While no definite signs of AMRO have been found, certain information about the FS geometry can be extracted from the magnetoresistance behaviour at $\theta$ very close to $90^{\circ}$. The main feature of the data around $\theta=90^{\circ}$ is a strong dependence of the resistivity on $\phi$. For $\theta=90^{\circ}$, this is illustrated in Figure 6a. This behaviour is consistent with the trigonal symmetry of the crystal (hexagonal in the layer plane) and is related to the geometry of the cross-section of the FS in the plane parallel to the layers, as described in reference [25]. The $\phi$-dependence of the interlayer resistivity is induced by the variation of the curvature of the FS cross-section: $R$ is expected to be minimum if the radius of curvature is maximum. The low temperature FS of the purple bronze is unknown. However, one can try to predict the geometry of the pockets of carriers left after the openings of density wave gaps, by analysing the normal state FS. Figure $6 \mathrm{~b}$ shows the calculated FS which includes three sheets corresponding to three $d$ sub-bands with strong nesting properties [6]. The unperfect nesting at the corners of the different sheets is due to the hybridization between the three $\mathrm{MoO}_{6}$ octahedra $1 \mathrm{D}$ chains. It is likely that the pockets left by the density wave gap openings are located at these corners. The observed $\phi$-dependence could be due to quasi-triangular pockets. On the other hand, it could also originate from small anisotropic pockets (for example strongly elongated in the plane) arranged in the Brillouin zone according to the hexagonal symmetry.

Another clear property of the curves of Figure 5 is the presence of a narrow peak centred at $\theta=90^{\circ}$. This appears on all curves, whatever $\phi$. The peak width slightly varies with $\phi$ and is independent of the field strength. This behaviour is characteristic of the so-called coherence peak, found in many organic conductors and attributed to a specific topology of electron orbits on a weakly warped cylindrical FS in a field nearly parallel to the layers [25]. The width of the coherence peak is directly determined by the warping of the FS cylinder through the relation: $\Delta \theta / k_{F} d=\Delta k_{/ /} / k_{F}$, where $\mathrm{d}$ is the interlayer distance (the $c$-parameter in the case of $\mathrm{KMo}_{6} \mathrm{O}_{17}, d=13.35 \AA$ ), $k_{F}$ is the in-plane Fermi wave vector and $\Delta k_{/ /}$measures the corrugation. In our case, the peak width $\Delta \theta$ is found to be close to $1^{\circ}$. The estimation of $k_{F}$ in the low temperature phase between $B_{2}$ and $B_{3}$ is more problematic. It can be evaluated only in low magnetic fields through Hall effect data [32]. An estimation of the carriers concentration had been performed with a two-band model with combined Hall effect, resistivity and magnetoresistance data. This analysis leads to an electron concentration at $4.2 \mathrm{~K}$ of the order of $6 \times 10^{18} \mathrm{~cm}^{-3}$. Assuming this carrier concentration is distributed into 6 equivalent quasi-cylindrical pockets, one can estimate in a free electron picture a rough order of magnitude for the Fermi wavevector. This yields a $k_{F}$ value of approximately $0.01 \AA^{-1}$, which should be compared to the extension of the pseudo-triangular parts of the normal state Fermi surface, centred at the corners of the Brillouin zone. ARPES measurements performed at room temperature give values close to $0.10 \AA^{-1}$ and $0.13 \AA^{-1}$ in the $\Gamma \mathrm{K}$ and KM directions [9] (Fig. 6b). The $k_{F}$ value of $0.01 \AA^{-1}$ for the pockets left at low temperature is therefore not unreasonable. With this value and an interlayer parameter $d=13.65 \AA$ (the $c$ parameter of the purple bronze), one obtains a corrugation parameter $\Delta k_{/ /} / k_{F}$ of he order of $10 \%$. This value is rather high, compared to quasi-2D organic conductors [25]. Nevertheless, it is obviously still small enough for the magnetoresistance to exhibit features typical of quasi-2D metals, as evidenced by the presence of the coherence peak. The corrugation parameter depends on the interlayer overlap integral $t_{\perp}$ through the relation $2 t_{\perp} / \varepsilon_{F}=\Delta k_{/ /} / 2 k_{F}$. However since the electron effective mass is unknown, it is not possible to evaluate the Fermi energy and therefore $t_{\perp}$.

One should note yet that the above result reveals a $3 \mathrm{D}$ FS open in the interlayer direction, but well defined in all directions, i.e. the transport across the layers is coherent. 


\section{Conclusion}

Interlayer magnetoresistance studies of the Mo purple bronze, $\mathrm{KMo}_{6} \mathrm{O}_{17}$, in the low temperature density wave state corroborate that this compound is a quasi twodimensional metal showing field induced transitions due to orbital effects. Many properties are similar to what is found in layered organic conductors. The angular properties at large polar angle $\theta$ are well accounted for by the existence of a quasi cylindrical warped Fermi surface, with a corrugation estimated to be of the order of $10 \%$. The anisotropy of the resistivity when $B$ is rotated in the layer plane is consistent with the trigonal symmetry. We note that the angular dependence of the magnetoresistance at small polar angle is not fully understood. Unlike in many other quasi-2D conductors, it is basically determined by only the out-of-plane field component; in particular it shows no sign of AMRO. Further work is necessary to clarify this behaviour.

The authors would like to thank Cécile Naud for providing the low temperature resistivity data $(T=150 \mathrm{mK})$. Part of this work is supported by Contract "Transnational AccessSpecific Support Action, RITA-CT-2003-505474" of the European Commission.

\section{References}

1. Low Dimensional Properties of Molybdenum Bronzes and Oxides, edited by C. Schlenker (Kluwer Acad. Publ., Dordrecht, 1989)

2. edited by M. Greenblatt, Oxide bronzes, Int. J. Mod. Phys. B 7, 4045 (1993)

3. Physics and Chemistry of Low-Dimensional Inorganic Conductors, edited by C. Schlenker, J. Dumas, M. Greenblatt, S. van Smaalen, NATO ASI Series B (Plenum, 1996), Vol. 354

4. R. Buder, J. Devenyi, J. Dumas, J. Marcus, J. Mercier, C. Schlenker, C. Schlenker, J. Phys. Lett. 43, L59 (1982)

5. H. Vincent, M. Ghedira, J. Marcus, J. Mercier, C. Schlenker, J. Sol. State Chem. 47, 113 (1983)

6. M.H. Whangbo, E. Canadell, C. Schlenker, J. Amer. Chem. Soc. 109, 6308 (1987); M.H. Whangbo, E. Canadell, P. Foury, J.P. Pouget, Science 252, 96 (1991); E. Canadell, M.H. Whangbo, in reference [2] p. 4005; E. Canadell, Chem. Mater. 10, 2770 (1998)

7. C. Escribe-Filippini, K. Konate, J. Marcus, C. Schlenker, R. Almairac, R. Ayroles, C. Roucau, Phil. Mag. B 50, 321 (1984)

8. G.H. Gweon, J.W. Allen, J.A. Clack, Y.X. Zhang, D.M. Poirier, P.J. Benning, C.G. Olson, J.Marcus, C. Schlenker, Phys. Rev. B 55, R13353 (1997)

9. H. Guyot, H. Balaska, P. Perrier, J. Marcus, Surf. Science 600, $3860(2006)$

10. M.A. Valbuena, J. Avila, V. Pantin, S. Drouard, H. Guyot, M.C. Asensio, Applied Surface Science, 252, 5415 (2006); M.A. Valbuena, J. Avila, S. Drouard, H. Guyot, M.C. Asensio, J. Phys. Chem. Solids 67, 213 (2006)

11. P. Mallet, K.M. Zimmermann, Ph. Chevalier, J. Marcus, J.Y. Veuillen, J.M. Gomez Rodriguez, Phys. Rev. B 60,
2122 (1999); J.Y. Veuillen, P. Mallet, L. Magaud, S. Pons, J. Phys.: Condens. Matter 15, S2547 (2003)

12. J. Dumas, E. Bervas, J. Marcus, D. Salomon, C. Schlenker, G. Fillion, J. Magn. Magn. Mat. 31-34, 535 (1983)

13. C. Schlenker, J. Dumas, C. Escribe-Filippini, M. Boujida, Physica Scripta 29, 55 (1989)

14. M. Tian, S. Hue, J. Shi, S. Li, Y. Zhang, J.Phys.: Condens. Matter 13, 311 (2001); M. Tian, S. Hue, S. Li, Y. Zhang, J. Appl. Phys. 89, 3408 (2001)

15. Y. Latyshev, A.A. Sinchenko, private communication; Y.I. Latyshev et al., in Recent Developments in Low Dimensional Charge Density Wave Conductors, Skradin (Croatia) June 29-July 3 (Publ. Institute of Physics, Zagreb, 2006)

16. J. Dumas, H. Guyot, H. Balaska, J. Marcus, D. Vignolles, I. Sheikin, A. Audouard, L. Brossard, C. Schlenker, Physica B 346-347, 314 (2004); see also A. Rötger, C. Schlenker, J. Dumas, J. Marcus, S. Dubois, A. Audouard, L. Brossard, J.P. Ulmet, S. Askenazy, Synth. Metals, 55-57, 2725 (1993); A. Rötger, Thesis, Université Joseph Fourier de Grenoble (1984)

17. H. Balaska, J. Dumas, H. Guyot, P. Mallet, J. Marcus, C. Schlenker, J.Y. Veuillen, D. Vignolles, Solid State Sciences 7, $690(2005)$

18. D. Zanchi, A. Bjelis, G. Montambaux, Phys. Rev. B 53, 1240 (1996); J. Phys. IV France 9, 203 (1999); P.D. Grigoriev, D.S. Lyubshin, Phys. Rev. B 72, 195106 (2005)

19. A.G. Lebed, JETP Lett. 78, 138 (2003)

20. P. Christ, W. Biberacher, M.V. Kartsovnik, E. Steep, E. Balthes, H. Weiss, H. Müller, JETP Lett. 71, 303 (2000)

21. N. Biskup, J.A.A.J. Perenboom, J.S. Qualls, J.S. Brooks, Solid State Commun. 107, 503 (1998)

22. C. Proust, A. Audouard, A. Kovalev, D. Vignolles, M. Kartsovnik, L. Brossard, N. Kushch, Phys. Rev. B 62, 2388 (2000)

23. D. Andres, M.V. Kartsovnik, W. Biberacher, H. Weiss, E. Balthes, H. Müller, N.D. Kushch, Phys. Rev. B 64, 161104(R) (2001); D. Andres, M.V. Kartsovnik, P.D. Grigoriev, W. Biberacher, H. Müller, Phys. Rev. B 68, 201101(R) (2003); M.V. Kartsovnik, D. Andres, W. Biberacher, C.R. Chimie 10, 6 (2007)

24. J. Wosnitza, Fermi Surfaces of Low-Dimensional Organic Metals and Superconductors (Springer-Verlag, Berlin Heidelberg, 1996)

25. M.V. Kartsovnik, Chem. Rev. 104, 5737 (2004)

26. H. Guyot, J. Dumas, J. Marcus, C. Schlenker, D. Vignolles, J. Phys. IV France 131, 261 (2005); J. Dumas et al., in Recent Developments in Low Dimensional Charge Density Wave Conductors, Skradin (Croatia) June 29-July 3 (Publ. Institute of Physics, Zagreb, 2006)

27. JETP 85, 337 (1997); V.G. Peschansky, Low Temp. Phys. 23, 35 (1997)

28. A.G. Lebed, N.N. Bagmet, Phys. Rev. B 55, R8654 (1997) 29. C. Marcenat, T. Klein, private communication.

30. L.P. Gorkov, A.G. Lebed, J. Phys. Lett. 45, L-433 (1984)

31. M. Héritier, G. Montambaux, P. Lederer, J. Phys. Lett. 45, L-94 (1984)

G. Montambaux, M. Héritier, P. Lederer, Phys. Rev. Lett. 55, 2078 (1985)

32. M. Boujida, C. Escribe-Filippini, K. Konaté, J. Dumas, G. Fourcaudot, C. Schlenker, Fizika (Zagreb) 21, 148 (1989); M. Boujida, Thesis, Université Joseph Fourier de Grenoble (1988)

33. Cécile Naud, private communication 